Draft VERSiOn SEPTEMBER 26, 2018

Preprint typeset using $\mathrm{LAT}_{\mathrm{E}} \mathrm{X}$ style AASTeX6 v. 1.0

\title{
IDENTIFYING RINGS IN IFU SURVEYS
}

\author{
CHIEN-HSiu $\operatorname{LEE}^{1}$ (李見修) \\ Subaru Telescope, NAOJ, 650 North A'ohoku Place, Hilo, HI 96720, USA
}

Keywords: Gravitational lensing: strong - Galaxies: elliptical and lenticular, cD

\section{INTRODUCTION}

According to general relativity (Einstein 1915), massive galaxies can induce strong space-time curvature, gravitationally focus the lights of background sources to form multiple images along the observer's line-of-sight. When the lens, source, and the observer are well aligned, the lensed image will become a ring, so-called Einstein ring (Einstein 1936). Despite their usefulness, only dozens of Einstein rings have been reported so far (see e.g. Bolton et al. 2008; Stark et al. 2013), limited by the low resolution images from ground-based telescopes. There were attempts to identify gravitational arcs using imaging (e.g. Paraficz et al. 2016) or spectroscopy (Bolton et al. 2008). However, these searches require extensive modeling and fitting. To minimize the efforts of finding heavily blended lens, we propose to identify strong gravitational lensing candidates using IFU surveys, with the aid of computer vision techniques.

\section{CONCEPT}

Because the space-time curvature is proportional to the underlying masses, gravitational arcs are more likely to be found around massive galaxies, i.e. luminous red galaxies (LRGs). However, these luminous galaxies often outshine the gravitational arcs. Indeed, it has been shown that there are numerous arcs heavily blended in the light profile of bright lenses, not resolvable from the poor spatial resolution imaging obtained by ground-based telescopes. One conventional method to identify heavily blended arcs is to search for emission features on top of the LRG spectrum. However, such approaches have only been applied to long-slit or single fiber spectra; thus can only identify possible candidates and still require high resolution imaging (e.g. from Hubble Space Telescope or ground-based telescopes with adaptive optics) to confirm the arcs. We propose to expand this approach to IFU surveys and reveal arcs in the spatially resolved spectra. Indeed, Smith (2017) has analyzed IFU spectra from MaNGA, and identified a strongly lensed system SDSSJ170124.01+372258.0 with dedicated modeling to remove the spectral contributions from the LRG. However, it is time consuming to model and remove LRG spectral contribution, hence Smith (2017) only analyzed 81 galaxies with $\sigma>250 \mathrm{~km} / \mathrm{s}$. Alternatively, we can use image subtraction to reveal arcs in the IFU data. As arcs have emission features on top of the LRG spectra, subtracting IFU images from subsequent wavelength will remove the LRG spectra and reveal arcs. After the arcs are revealed, we can adopt computer vision techniques, e.g. the Hough transform (Hough 1962; Duda \& Hart 1972), to replace human inspection and automatically identify the arc patterns, as demonstrated in Lee (2017).

\section{APPLICATION}

As a proof of concept, we used SDSSJ170124.01+372258.0 to demonstrate the feasibility of the approach mentioned in the previous section. We acquired its MaNGA IFU spectra from SDSS SkyServer ${ }^{1}$, which covers wavelength from 3622 to $10353 \AA$ and a spectral resolution of $1 \AA$. We employed the IRAF $^{2}$ routine imslice to extract $2 \mathrm{D}$ IFU images from the 3D IFU data-cube (see Fig. 1). We then used the IRAF routine imarith to subtract the 2D IFU images. Examples of IFU images at a given wavelength and lensed arcs from subtracted images can be found in Fig. 1. We then applied Hough transform algorithm to the MaNGA $6676 \AA$ - $6675 \AA$ IFU image, where the [OII] emission from the background source form an arc pattern (Fig. 1). It only takes 0.13 seconds for Hough transform to identify this arc pattern on a $2.7 \mathrm{GHz}$ Intel Core i5 processor.

For a single object, it takes 20 seconds to download the MaNGA data, 5 seconds to extract 2D IFU images from the 3D data-cube, 15 seconds for image subtraction, and 875 seconds for Hough transform to analyze all subtracted

\footnotetext{
${ }^{1}$ https://skyserver.sdss.org/dr13/en/tools/chart/charthome.aspx

2 http://iraf.noao.edu
} 

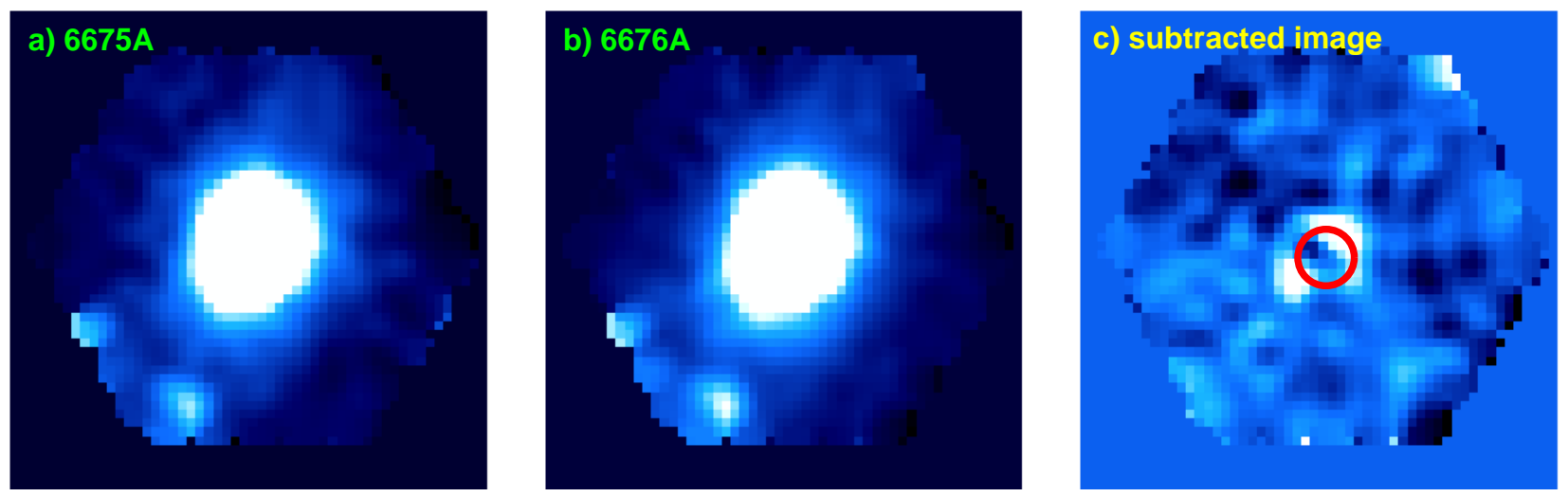

Figure 1. MaNGA data of SDSS J170124.01+372258.0. a) IFU image at $6675 \stackrel{\AA}{A}$. b) IFU image at $6676 \stackrel{\AA}{A}$. c) subtracted image from b) - a). The arc pattern identified by the circular Hough transform is marked in the red circle.

images. To summarize, this only costs $\sim 15$ minutes to fully analyze one single object.

\section{PROSPECTS}

Using the recently discovered MaNGA lens by Smith (2017), we demonstrate the feasibility of an automated approach to identify strong gravitational lenses in IFU surveys. This approach is applicable to other IFU surveys, e.g. CALIFA, SAMI, Hector, DESI, and 4MOST, which will all deliver IFU data-cube in similar format as MaNGA. The arc identification procedure can be improved with more realistic lens modeling, e.g. ARCFINDER (Alard 2006) for arcs, or CHITAH (Chan et al. 2015) for multi-imaged quasars, at the cost of computation time. Nevertheless the Hough transform approach (as proposed here) already provides a simple solution to detect arc patterns in a timely manner for the big data to come.

\section{REFERENCES}

Alard C., 2006, astro, arXiv:astro-ph/0606757

Bolton A. S., et al., 2008, ApJ, 682, 964-984

Chan J. H. H., et al., 2015, ApJ, 807, 138

Duda R. O., Hart P. E., 1972, Comm. ACM, 15, 11

Einstein A., 1915, SPAW,

Einstein A., 1936, Sci, 84, 506

Hough P.V.C., 1962, U.S. Patent 3068654
Lee, C.-H. 2017, PASA, 34, 014

Paraficz D., et al., 2016, A\&A, 592, A75

Smith R. J., 2017, MNRAS, 464, L46

Stark D. P., et al., 2013, MNRAS, 436, 1040 